\title{
Editorial
}

\section{Advocacy in Neonatology}

\author{
Avroy A. Fanaroff, MD ${ }^{1,2}$ Jonathan M. Fanaroff, MD, JD \\ ${ }^{1}$ Department of Pediatrics, Case Western Reserve University School \\ of Medicine, Cleveland, Ohio \\ 2 Division of Neonatology, Rainbow Babies and Children's Hospital, \\ Cleveland, Ohio \\ 3 The Rainbow Center for Pediatric Ethics, Rainbow Babies and Children's \\ Hospital/University Hospitals Health System, Cleveland, Ohio
}

Am J Perinatol 2019;36(suppl S2):S9-S12.

\section{Advocating for Preterm Infants}

Advocating for equality for preterm infants is complex, difficult, soul searching, must not be discriminatory, and requires a highly trained, compassionate, and caring professional team working in tandem with the family. At the borders of viability, currently designated the periviable period, ${ }^{1,2}$ there is considerable uncertainty regarding the risks to mother and infant regarding mortality and morbidity. Although there is abundant data concerning neonatal morbidity and mortality, the evidence is valid for a group, but unreliable for a given individual. Of concern in evaluating the available outcome data is that it is mainly reported by gestational age and this number is so imprecise. ${ }^{3}$ Even with early ultrasound evaluation the margin of error exceeds a whole week which at gestational ages between 22 and 25 weeks is huge and complicates the team's decision regarding active intervention. Furthermore, there is considerable difference in outcomes between 24 weeks and 3 days and 24 weeks and 6 days. Both groups being considered -24 weeks.

Neonatal intensive care units (NICUs) aim to provide safe, high-quality medical and surgical care for all newborns. The creation of, and widespread participation in, NICU quality collaboratives has no doubt accelerated progress in the field of neonatal quality improvement and safety. However, there still remains limited evidence of overall efficacy of these collaboratives. NICUs strive to apply the best possible evidence in their local setting, known as "potentially better practices," to achieve improved patient outcomes. However, similar interventions applied in a similar fashion in similar NICUs often do not produce the same results. This unexplained variability in outcomes between NICUs begs the question: What is the secret sauce? Why do some NICUs consistently outshine others in spite of the application of the same "potentially best practices"? To answer these questions, it becomes necessary to determine those factors that contribute to success or failure of improvement programs, including less tangible aspects such as NICU culture/environment, leadership, teamwork, and organizational structure. Only when these questions are satisfactorily answered can we anticipate more consistent short- and longterm outcomes. Evaluating specific care practices may illuminate key factors.

\section{Skin-to-Skin Care}

In the 1970s, kangaroo mother care (KMC) was developed in Columbia as a new way of caring for low birth-weight infants: in comparison with the usual cautious practice of incubator nursing with severely restricted parental access and discharge only when a weight of $1,700 \mathrm{~g}$ was attained, KMC involved "strapping the baby upright to the mother's chest in skin-to-skin contact, frequent (exclusive or nearly exclusive) breast feeding, formula supplements if weight gain did not exceed $20 \mathrm{~g} /$ day, and early discharge. A metaanalysis of 988 infants enrolled in three randomized controlled trials of continuous KMC begun in the first postnatal week in low- or middle-income countries found a $51 \%$ reduction in mortality among infants with a birth weight $<2,000$ g (relative risk: 0.49 ; $95 \%$ confidence interval [CI]: $0.29-0.82$ ). Since then, KMC in its original and newer flavors has been integrated into the mainstream of newborn care. Variations of KMC involving briefer periods of infant holding by the mother or father, appropriately called skin-to-skin care (SSC), are practiced in birthing hospitals in high-, low-, and middle-income countries in support of the Baby-friendly Hospital Initiative Step 4 ("help mothers initiate breastfeeding within 1 hour of birth). A 2016 Cochrane review ${ }^{4}$ examined 21 studies including 3,042 infants and concluded that KMC for (stabilized) low birth-weight infants in low-
Address for correspondence

Avroy A. Fanaroff, MD, PhD, Case

Western Reserve University

School of Medicine, Emeritus Eliza

Henry Barnes Professor in

Neonatology, Rainbow Babies and

Children's Hospital, Cleveland,

$\mathrm{OH}$, United States

(e-mail: aaf2@case.edu).
Copyright (C) 2019 by Thieme Medical Publishers, Inc., 333 Seventh Avenue, New York, NY 10001, USA. Tel: +1(212) 584-4662.
DOI https://doi.org/ 10.1055/s-0039-1691776. ISSN 0735-1631. 
resource settings was preferable to conventional neonatal care, with lower risk of death and severe infection and higher rates of breastfeeding at term postmenstrual age or discharge from the hospital.

The most substantial evidence of benefit from SSC is for breastfeeding. Intermittent SSC is associated with longer and more exclusive breastfeeding and higher volumes of expressed milk. SSC improves maternal attachment, promotes the participation of the mother and father in the infant's care, strengthens the family role in the care of a fragile infant, and decreases feelings of helplessness.

SSC has also been advocated for the nonpharmacologic management of procedural pain, e.g., heel lance. SSC is to be advocated on behalf of stable preterm infants and even for brief periods for technology-dependent babies.

\section{Vaccinations at Birth}

Immunization is one of the most cost-effective and lifesaving interventions preventing over 2.5 million deaths annually. Unfortunately over 20 million children living in underdeveloped countries still do not have access to vaccines or are under-immunized. Hepatitis B is a chronic infection that ultimately leads to cirrhosis, liver failure, and hepatic carcinoma. Mothers can pass the hepatitis B virus to their baby during childbirth without knowing it, since it is passed through blood and bodily fluids.

Immunization is very effective, protects the baby from hepatitis $B$, and protects others from the disease because children with hepatitis B usually are asymptomatic but may infect through contacts.

The vaccine protects the child from developing liver disease and cancer. The Centers for Disease Control and Prevention $(C D C)^{5}$ recommends three doses of the hepatitis $B$ shot for best protection at each of the following ages.

Shortly after birth, one through 2 months, and six through 18 months.

The hepatitis B vaccine is very safe, and it is effective in preventing hepatitis B. Greater efforts need to be made to ensure that this vaccine be available throughout the world. Moturi et $\mathrm{al}^{6}$ reported that staff perceptions, lack of daily vaccine availability, and lack of outreach programs to reach babies born outside health facilities with essential services are barriers for implementing timely delivery of hepatitis B birth dose (HepB-BD) vaccine. Addressing these challenges may accelerate HepB-BD implementation in Africa.

\section{Quality Improvement Worldwide}

An estimated 2.6 million newborns died in 2016; over 98.5\% of deaths occurred in low- and middle-income countries. Neonates born preterm and small for gestational age are particularly at risk given the high incidence of infectious complications and cardiopulmonary and neurodevelopmental disorders in this group. ${ }^{7}$

Quality improvement initiatives can reduce the burden of mortality and morbidity for hospitalized newborns in these settings. A prime example relates to central line-associated blood stream infections (CLABSIs), the most common complication of central catheters in neonates. These infections increase length of hospital stay, hospital costs, and impact on mortality and morbidities. Taylor et al's quality improvement initiative ${ }^{8}$ using checklists, supported with education and feedback, significantly reduced CLABSIs in their neonatal unit by $41 \%$, from 13.8 definite CLABSIs per 1,000 central-line days to 7.8 definite CLABSIs per 1,000 central-line days. ${ }^{8}$ Central-line venous catheters have permitted lifesaving treatment for critically ill neonates; however, the attributable mortality rate of CLABSIs has been estimated to be between 4 and $20 \%$.

For decades the prevailing thinking was that CLABSIs and nosocomial infections were inevitable and unavoidable. The mindset now is that each infection reflects a failure and should have been avoided.

A comprehensive catheter-related staff education, sterile surgical like preparation and dress for line placements, standardization of skin preparation, introduction of new antiseptic agents, implementation of central catheter insertion and maintenance checklists, reinforcement of the use of maximal sterile barrier precautions, and revision of the central catheter configuration and maintenance protocols including minimal invasion of the line are the key components of CLABSI protocols.

The application of the tools of quality improvement during the last decade has resulted in a dramatic decline in these infections. Other successful programs relate to avoiding hypothermia in the delivery room, quality improvement in developing countries, and the management of jaundice. ${ }^{9-11}$

Quality improvement efforts have implications for racial equality. A study of 743 NICUs in the Vermont Oxford Network found not only that black, Hispanic, and Asian infants were segregated across NICUs, but also that, compared with white infants, black infants received care at lower-quality NICUs. ${ }^{12}$

\section{Vitamin K}

Vitamin $\mathrm{K}$ administration at birth enhances the clotting mechanism (factors V, VII, IX, and X) and prevents serious bleeding. It is administered intramuscularly in the United States, but orally in many parts of Europe where a second and a third oral dose is necessary. The vitamin K administration can prevent potentially fatal intracranial and gastrointestinal bleeding due to hemorrhagic disease of the newborn, now designated as Vitamin K deficiency bleeding (VKDB). A onetime prophylactic intramuscular (IM) injection of vitamin $\mathrm{K}$ administered shortly after birth, recommended by the American Academy of Pediatrics (AAP) since 1961, had virtually eliminated this serious disease. ${ }^{13}$ In recent years in the United States, there has been an increase in the number of reports of parents who refuse IM vitamin $K$ for their newborns with a concomitant increase in reports of newborns with VKDB. ${ }^{14}$

In their study, Loyal et $\mathrm{al}^{15}$ determined that $0.6 \%$ of parents in the United States refuse to accept this prophylactic therapy for their newborns. Regional rates vary between 0.5 and $2.3 \%$. Exclusive breastfeeding, non-Hispanic white race 
and/or ethnicity, female gender, gestational age, and mothers were significantly associated with refusal of IM vitamin $\mathrm{K}$ administration. Refusal of the administration of both ocular prophylaxis and hepatitis B vaccine was also strongly associated with refusal of IM vitamin K administration. "Refusal of IM vitamin $\mathrm{K}$ by parents of newborns is a significant problem. Interventions to minimize risks to these newborns are needed." 15

\section{Screening for Critical Congenital Heart Disease}

An expert panel concluded that incorporation of pulse oximetry to the assessment of the newborn infant can enhance detection of critical congenital heart disease (CCHD). In 2012, the Secretary of Health and Human Services (U.S. Department of Health and Human Services) recommended that screening for CCHD be added to the uniform screening panel. ${ }^{16}$ The AAP then became a strong advocate and driving force for early detection of CCHD. They published strategies for the implementation of pulse oximetry screening, including equipment and training of personnel, important training addressing the interpretation of the oximetry findings, and appropriate follow-up of positive results. Similar programs have been implemented in the United Kingdom, Sweden, Italy, and other parts of Europe. The results have been spectacularly good and have saved many lives as noted in Plana et al's Cochrane review. ${ }^{17}$

Nineteen studies provided data for the primary analysis (oxygen threshold $<95 \%$ or $\leq 95 \%$; $N=436,758$ participants). The overall sensitivity of pulse oximetry for the detection of CCHD was 76.3\% (95\% CI: 69.5-82.0). Specificity was $99.9 \%$ (95\% CI: 99.7-99.9), with a false-positive rate of 0.14\% (95\% CI: $0.07-0.22$ ) (high certainty of the evidence). These results showed that out of 10,000 apparently healthy late preterm or full-term newborn infants, six will have CCHD. Screening by pulse oximetry will detect five of these infants as having CCHD and will miss one case. In addition, screening by pulse oximetry will falsely identify another 14 infants out of the 10,000 as having suspected CCHD when they do not have it. The false-positive rate for the detection of CCHD was lower when newborn pulse oximetry was performed $>24$ hours after birth than when it was performed within 24 hours. However, many of the false positives have respiratory disorders including pneumonia and transient tachypnea of the newborn or sepsis, so the screening aided in the early detection of these disorders. In summary pulse oximetry is a highly specific and moderately sensitive test for the detection of CCHD with very low false-positive rates. It should be an integral part of all newborns' evaluation prior to discharge from hospital. Current evidence supports the introduction of routine screening for CCHD in asymptomatic newborns before discharge.

\section{Human Milk Feeding}

There is now compelling data that nutritional inadequacies during fetal life and the first 2 years after birth correlate negatively with the child's neurodevelopment mandates advocating for nutritional support and education during pregnancy and early childhood. Furthermore, nutritional deficiencies during this critical time period may result not only in lifelong brain dysfunction but also other long-term problems including obesity, hypertension, and diabetes. Key nutrients that support optimal brain development include protein, zinc, iron, choline, folate, iodine, vitamins $A, D, B_{6}$ and $\mathrm{B}_{12}$, and long-chain polyunsaturated fatty acids. All providers caring for mothers and children must advocate for healthy diets for mothers, infants, and young children so that they lay the foundation for optimum brain development in the first 1,000 days. ${ }^{18}$

Citing "breast milk is the perfect food," health care professionals, consumer advocacy groups, and the AAP have been encouraging new mothers to exclusively breastfeed. ${ }^{19}$

The benefits of human milk are numerous and include psychological, convenience, economical, ecological, and nutritional superiority, so that exclusively breast fed infants are protected from many illnesses. However, the composition and calorie density of human milk varies enormously. Human milk is a composite of nutritional choices of the mother, commencing in the preconceptual era. Maternal food choices affect the nutritional profile of human breast milk. Overall, human breast milk has been found to be low in certain nutrients in developed countries: vitamin $\mathrm{D}$, iodine, iron, and vitamin $\mathrm{K}$. Additional nutrient deficiencies have been documented in resource-poor countries: vitamin $\mathrm{A}$, vitamin $\mathrm{B}-12$, zinc, and vitamin B-1/thiamin. Despite these deficiencies it is the milk of choice and the availability of donor human milk and the ability to fortify the human milk have facilitated the provision of exclusive human milk diet to even the most immature preterm infants with clear-cut evidence of benefit to the baby. Even partially fed infants benefit as Lechner and $\operatorname{Vohr}^{20}$ have demonstrated a direct correlation between intelligence and the amount of human milk consumed. The components of breast milk thought to mediate improved cognitive outcomes include long-chain polyunsaturated fatty acids and human milk oligosaccharides. So advocates should continue to support exclusive human milk feeding for all babies with the exception of those with inherited metabolic conditions who would be harmed by the milk.

\section{Conclusion}

In summary there are many opportunities to advocate on behalf of our neonatal patients. Whereas the overall results of neonatal intensive care have been excellent, there remain huge discrepancies in major outcomes, morbidity, and mortality within a country, between countries, and by race. Not only should evidence-based medicine and where possible personalized medicine be practiced, but also remedies should be urgently sought to ensure optimal outcomes for all babies. All means including the social media should be utilized to advocate for these babies.

Conflict of Interest

None declared. 


\section{References}

1 Mercer BM. Periviable birth and the shifting limit of viability. Clin Perinatol 2017;44(02):283-286

2 Batton D, Batton B. Advocating for equality for preterm infants. CMAJ 2013;185(18):1559-1560

3 Helenius K, Sjörs G, Shah PS, et al; International Network for Evaluating Outcomes (iNeo) of Neonates. Survival in very preterm infants: an international comparison of 10 national neonatal networks. Pediatrics 2017;140(06):e20171264

4 Moore ER, Bergman N, Anderson GC, Medley N. Early skin-to-skin contact for mothers and their healthy newborn infants. Cochrane Database Syst Rev 2016;11:CD003519

5 Centers for Disease Control and Prevention. Monthly MMWR, April 2018. Available at: https://www.cdc.gov/mmwr/index2018. html. Accessed May 7, 2019

6 Moturi E, Tevi-Benissan C, Hagan JE, et al. Implementing a birth dose of hepatitis B vaccine in Africa: findings from assessments in 5 countries. J Immunol Sci 2018Suppl(5):31-40

7 Zaka N, Alexander EC, Manikam L, et al. Quality improvement initiatives for hospitalised small and sick newborns in low- and middle-income countries: a systematic review. Implement Sci 2018;13(01):20

8 Taylor JE, McDonald SJ, Earnest A, et al. A quality improvement initiative to reduce central line infection in neonates using checklists. Eur J Pediatr 2017;176(05):639-646

9 Chawla D, Suresh GK. Quality improvement in neonatal care - a new paradigm for developing countries. Indian J Pediatr 2014;81 (12):1367-1372

10 Russo A, McCready M, Torres L, et al. Reducing hypothermia in preterm infants following delivery. Pediatrics 2014;133(04):e1055-e1062

11 Tartaglia KM, Campbell J, Shaniuk P, McClead RE. A quality project to improve compliance with AAP guidelines for inpatient man- agement of neonatal hyperbilirubinemia. Hosp Pediatr 2013;3 (03):251-257

12 Howell EA, Hebert PL, Zeitlin J. Racial segregation and inequality of care in neonatal intensive care units is unacceptable. JAMA Pediatr 2019;173(5):420-421

13 American Academy of Pediatrics Committee on Fetus and Newborn. Controversies concerning vitamin $\mathrm{K}$ and the newborn. Pediatrics 2003;112(1, Pt 1):191-192

14 Centers for Disease Control and Prevention (CDC). Notes from the field: late vitamin $\mathrm{K}$ deficiency bleeding in infants whose parents declined vitamin K prophylaxis-Tennessee, 2013. Morb Mortal Wkly Rep 2013;62(45):901-902

15 Loyal J, Taylor JA, Phillipi CA, et al; BORN investigators. Factors associated with refusal of intramuscular vitamin $\mathrm{K}$ in normal newborns. Pediatrics 2018;142(02):142

16 Mahle WT, Martin GR, Beekman RH 3rd, Morrow WR; Section on Cardiology and Cardiac Surgery Executive Committee. Endorsement of health and human services recommendation for pulse oximetry screening for critical congenital heart disease. Pediatrics 2012;129:190-192

17 Plana MN, Zamora J, Suresh G, Fernandez-Pineda L, Thangaratinam S, Ewer AK. Pulse oximetry screening for critical congenital heart defects. Cochrane Database Syst Rev 2018;3:CD011912

18 Schwarzenberg SJ, Georgieff MK; COMMITTEE ON NUTRITION. Advocacy for improving nutrition in the first 1000 days to support childhood development and adult health. Pediatrics 2018;141 (02):e20173716

19 Erick M. Breast milk is conditionally perfect. Med Hypotheses 2018;111:82-89

20 Lechner BE, Vohr BR. Neurodevelopmental outcomes of preterm infants fed human milk: a systematic review. Clin Perinatol 2017; 44(01):69-83 